\title{
Beeswax-Lecithin SLMs of Erythromycin Ethyl Succinate for Treatment of Acute Bacteremia in Infected Mice
}

\author{
Uduma Eke Osonwa $^{1}$, Onyinye Uwaezuoke ${ }^{2}$ and Ngozi Ilunoh ${ }^{1}$ \\ 1. Department of Pharmaceutics and Pharmaceutical Technology, Faculty of Pharmaceutical Sciences, Nnamdi Azikiwe University, \\ Awka, Anambra State, Nigeria \\ 2. Department of Pharmaceutics and Pharmaceutical Technology, Faculty of Pharmacy, Ogun State University, Ago-Iwoye, Ogun
} State, Nigeria

\begin{abstract}
The aim of this work was to increase the efficacy of erythromycin ethyl succinate by encapsulation in beeswax lipid matrix using Myrj 52 as emulsifier. Different batches of SLM's (solid-lipid microparticles) were formulated and stable ones were selected. The encapsulation efficiency and loading capacities were calculated. The batch with the highest loading capacity was used for further assays. The particle size was determined by light microscopy. The sensitivity of different clinical bacterial isolates to erythromycin was tested using in vitro cultures and $E$. coli was selected for efficacy tests. The activity of the formulated drug was tested in the in vitro culture and compared to that of the unformulated drug. White albino mice were infected with $E$. coli and left for one day to develop significant bacteremia. They were then divided into 4 groups $(n=4)$ and treated with the formulation and unformulated drug at a dose of $7.14 \mathrm{mg} / \mathrm{kg} 8$ hourly for 56 hours. A third group was given SLM's that do not contain drug, while another group was left untreated. The selected batch has an encapsulation efficiency of $94.83 \%$ with a loading capacity of $3.88 \%$. The particle size was $17 \pm 4 \mu \mathrm{m}$. At the end of the three day period of treatment, the group treated with unformulated erythromycin had much stooling and weakness in the mice, and some deaths were recorded, while that treated with the formulation had $33.8 \%$ bacteremia and the clinical signs had largely subsided. The other two groups recorded deaths the following day after bacteremia induction. The results show marked improvement in efficacy of erythromycin ethyl succinate by formulation in SLMs with beeswax and lecithin as lipid matrix.
\end{abstract}

Key words: Erythromycin, SLM, beeswax-lecithin SLM, E. coli, improved activity.

\section{Introduction}

Automated combinatorial chemistry has made drug design and synthesis less complex leading to an unprecedented turn over in the volume of new chemical entities, a great majority of which are poorly soluble BCS class II drugs [1]. It has been postulated that approximately $70 \%$ of discovery compounds and $40 \%$ of pipeline candidates are insoluble in water and consequently have poor bioavailability [2] and may not reach the market [3]. Formulation scientists are constantly searching for better ways of delivering

Corresponding author: Uduma Eke Osonwa, $\mathrm{PhD}$, research field: drug delivery in tropical diseases. these entities in a manner that not only improves their aqueous solubility and consequently bioavailability but also reduces their side effect. Older, established therapeutic agents with various delivery limitations are also finding a new lease on life through these newer drug delivery systems. In recent times, scientists have turned to lipids in their quest for solution.

Lipid-based drug delivery systems represent a class of physiologically well-tolerated formulation whose most frequent role is to improve the solubility of poorly soluble drugs with particular emphasis on BCS class II and IV [4]. They have been shown to improve bioavailability through a rare combination of 
solublization, dispersion or encapsulation, and stimulation of the digestive process as well as potential inhibition of the receptor mediated efflux or pre-systematic metabolism or passage through the lymphatic system [5]. Lipid based drug delivery systems come in a variety of presentations and include solutions, suspensions, emulsions, microemulsions, SEDDS (self-emulsifying drug delivery systems), SMEDDS (self microemulsifying drug delivery systems), SNEDDS (self-nanoemulsifying drug delivery systems), dry emulsions, SLMS (solid lipid microparticles), and SLNs (solid lipid nanoparticles) [6].

SLMs (solid lipid micro particles) are particles of micrometer dimensions with a diameter ranging from 1 to $1,000 \mu \mathrm{m}$ made up of solid lipid matrix. They are oily droplets made from polymeric, waxy or modified natural polymers like starches, gums, proteins and others which are lipids, solid at room temperature and stabilized by surfactants [7]. In spite of some challenges of SLMs mostly poor drug loading and particle growth [8], they still offer a wide range of possibilities to the formulation scientists. These include enhancement of the bioavailability of poorly water soluble molecules [9] protection of sensitive drug molecules from the outer environment (water, light) and even controlled release characteristics using biodegradable physiological lipid that decrease the danger of acute and chronic toxicities while avoiding the use of organic solvents $[10,11]$ controlling and targeting the release of incorporated medicinal agents [12].

Erythromycin is the first macrolide antibiotic isolated over six decades ago from a strain of Saccharopolyspora erythraea (formerly Streptomyces erythraeus). The base, stearate salt and the esters are poorly water-soluble. In addition, they are unstable at the acidic gastric $\mathrm{pH}$ and hence irregularly absorbed [13]. A variety of preparations have been developed in an effort to improve bioavailability and make absorption more predictable (enteric- and "film"-coated tablets of the base; enteric-coated pellets of the base in capsules; the stearate salt in "film"-coated tablets; the ethyl succinate ester available in tablet or oral suspension; the estolate in tablets, capsules or oral suspensions) [14] yet erythromycin bioavailability remains formulation dependent, highly variable and erratic [15].

Many of the drawbacks of parenteral administration including extravasation of drug or blood, catheter infection, thrombosis and over expression of side effects are some of the reasons for the preference of the oral route till date. The oral route remains the route of choice for most ailments [16]. For erythromycin the goals of formulation will be to overcome some of its challenges when administered orally and these may include instability at acid $\mathrm{pH}$, short elimination half-life, and poor digestive tolerance [12]. The goal of the present work will therefore be to improve the oral bioavailability of erythromycin ethyl succinate by formulating it as solid lipid microparticles.

\section{Materials and Methods}

\subsection{Reagents and Laboratory Animals}

\subsubsection{Materials}

Erythromycin (ethyl succinate) was a generous gift from Juhel (Pharma) Nigeria Ltd. Myrj52 and chloroform were obtained from Sigma Aldrich. Lecithin and beeswax were gifts from the Department of Pharmaceutics and Pharmaceutical Technology, Nnamdi Azikiwe University. All other materials were of analytical grade and used without further purification.

\subsubsection{Laboratory Animals}

Sixteen (16) white albino mice of both sexes weighing 22-25 g were acquired from the Department of Pharmacology and Toxicology, Faculty of Pharmaceutical Sciences of Nnamdi Azikiwe University, Awka, Nigeria. The animals were used within the animal facilities and so needed only one week of acclimatization. All animals were used in accordance with the approval of the Animals Ethics Committee of the Faculty. 


\subsection{Preparation of Erythromycin Loaded Solid-Lipid Microparticles}

Erythromycin-SLMs were prepared using the emulsification solvent evaporation technique. Beeswax, erythromycin, lecithin and Myrj $52^{\circledR}$ were dissolved in chloroform in a beaker. Distilled water was gradually added to the mix. The resulting solution was heated to $75 \pm 2{ }^{\circ} \mathrm{C}$ with stirring at $1,000 \mathrm{rpm}$ on a hot plate. The stirring continued for $3 \mathrm{~h}$, allowing enough time for the organic solvent to completely evaporate and the volume of the mixture to condense to approximately $5 \mathrm{~mL}$. The flask was then removed from the hot plate, and $10 \mathrm{~mL}$ of ice-cold $\left(0-2{ }^{\circ} \mathrm{C}\right)$ water was used to reconstitute the mixture which was then stirred again at $1,000 \mathrm{rpm}$ for $2 \mathrm{~h}$. The resulting suspension was centrifuged in a refrigerated centrifuge (Eppendorf 5804R) at 5,000 rpm and $4{ }^{\circ} \mathrm{C}$ for $2 \mathrm{~h}$ to isolate and subsequently remove the supernatant. The composition of the various formulations is outlined in Table 1 .

\subsection{Characterization of Solid Lipid Microparticles}

\subsubsection{Physical Properties Assessment}

The various formulations of erythromycin SLMs were visually assessed after production and at various time intervals afterwards for clarity, color, odor and homogeneity. A portion of each formulation was stored at room temperature and another portion at $4{ }^{\circ} \mathrm{C}$ for 72 hours.

\subsubsection{Particle Size and Size Distribution}

The particle size and size distribution of the selected formulations were determined by light microscopy using a photomicrograph.

\subsubsection{Entrapment Efficiency and Loading Capacity}

The entrapment efficiency of each formulation was determined indirectly by measuring the concentration of the free unloaded drug in the supernatant after centrifugation of the microparticles. A $100 \mathrm{mg}$ sample of the erythromycin SLMs was dissolved in $100 \mathrm{~mL}$ of $70 \%$ ethanol to give a $1 \mathrm{mg} / \mathrm{mL}$ solution that was centrifuged in an Eppendorf 5804R refrigerated centrifuge tube at about 2,000 rpm for 1 hour. A $1 \mathrm{~mL}$ volume of the supernatant was diluted 1,000 fold using distilled water. The absorbance was determined using UV-spectrophotometer (Jenway 6505) at 360 $\mathrm{nm}$. The drug EE (entrapment efficiency) was calculated using Eq. (1):

$$
E . E=\frac{\text { Total amount of drug added-amount of unencapsulated drug }}{\text { Total amount of drug added }} \times 100
$$

The loading capacity is usually expressed as the ratio of the weight of entrapped drug to the total weight of lipid expressed as a percentage. The formula is given in Eq. (2).

$$
\text { Loading capacity }=\frac{W d}{W} \times 100
$$

$W d$ is entrapped drug in the lipid;

$W$ is the total weight of the particles in the formulation.

\subsection{In vitro Assessment of Antimicrobial Efficacy}

The agar well diffusion method was used to determine the inhibition zone diameter for the formulation with the best entrapment efficiency and the result compared with that of the unformulated drug at different concentrations. The SLMs without drug was also used as a control.

\subsection{In Vivo Efficacy Study}

Four groups made up of four mice each were used for this study. The first group of mice received the pure drug, the second group received erythromycin SLMs, the third group received plain SLMs, and the fourth group were untreated while the last group received only sterile water. Animals in groups 1, 2, 3, and 4 were infected with E. coli $\left(10^{4}\right.$ McFarland standards) by intra peritoneal injection. Sample blood was collected 24 hours after infection by tail milking of the albino rats. The sample was then cultured in nutrient broth for bacterial resuscitation for 2 days. 
Table 1 Composition of the various batches of the solid lipid microparticles.

\begin{tabular}{lllll}
\hline Batch & Erythromycin $(\mathrm{mg})$ & Beeswax $(\mathrm{g})$ & Myrj 52 $(\mathrm{g})$ & Lecithin $(\mathrm{g})$ \\
\hline A & 100 & 0.5 & 1.5 & 0.5 \\
$\mathrm{~B}$ & 100 & 0.5 & 1.5 & 0.25 \\
$\mathrm{C}$ & 100 & 0.25 & 1.5 & 0.25 \\
$\mathrm{D}$ & 100 & 0.25 & 1.5 & 0.5 \\
E & 100 & 0.5 & 1.5 & 0.125 \\
\hline
\end{tabular}

Baseline blood counts of the organism were obtained from the animals by colony counting technique using McConkey Agar prior to treatment which was initiated 24 hours after infection. The agar was prepared according to manufacturing specification. The animals in the groups were treated at intervals of eight hours for a total of 72 hours. The dose of erythromycin administered both for formulation and unformulated drug was $7.14 \mathrm{mg} / \mathrm{kg}$. Physical changes in the animals were noted and the final blood count taken.

\section{Results}

\subsection{Physical and Stability Assessment}

On visual inspection, the solid lipid microparticles appeared milky and translucent with a yellowish white color. Some of the formulations separated after storage at room and refrigerated temperatures but formulations $\mathrm{B}$ and $\mathrm{C}$ remained stable and maintained their homogeneity throughout the period of the research.

\subsection{Characterization of Solid lipid Microparticles}

The result of the characterization of the solid lipid microparticles is shown in Table 2.

\subsection{Particle Size}

The formulation which had the best entrapment efficiency was chosen and the particle size determined using the photomicrograph (Fig. 1). The mean particle size of the chosen formulation was $17 \pm 4 \mu \mathrm{m}$.

\subsection{In Vitro Antimicrobial Assay}

The inhibition zone diameter for the pure erythromycin sample, erythromycin SLMs and plain SLMs is shown in Tables 3, 4, and 5, respectively.

\subsection{In Vivo Effect on Bacteremia}

Table 6 shows the results of the study of the in vivo outcomes of the various formulations in mice.

\section{Discussion}

The various batches of the SLMs were formed with varying degrees of physical stability.

Some of the formulations separated hours after preparation and were excluded. Batches $\mathrm{B}$ and $\mathrm{C}$ remained stable but batch $B$ was selected for further work because of its higher entrapment efficiency. The mean particle size of the selected formulation was 17 $\pm 4 \mu \mathrm{m}$. Drug entrapment efficiency is usually a useful tool when drug carrier systems are evaluated and depicts the ability of the carrier system to solubilize the poorly soluble drug in its core. Method of incorporation and the nature of interaction between the model drug and the encapsulating matrix are some of the factors that affect $\mathrm{EE} \%$. It can be seen from Table 2 that the various formulations studied were

Table 2 Encapsulation efficiency and loading capacity.

\begin{tabular}{lccll}
\hline Batch & $\begin{array}{l}\text { Amount } \\
(\mathrm{mg})\end{array}$ & $\begin{array}{c}\text { unentrapped Amount entrapped } \\
(\mathrm{mg})\end{array}$ & $\begin{array}{l}\text { Encapsulation efficiency Drug } \\
(\%)\end{array}$ & $\begin{array}{l}\text { loading } \\
(\%)\end{array}$ \\
\hline A & 11.01 & 88.99 & 88.99 & 3.31 \\
B & 5.17 & 94.83 & 94.83 & 3.88 \\
C & 18.97 & 81.21 & 81.21 & 3.76 \\
D & 21.60 & 78.40 & 78.40 & 3.21 \\
E & 19.13 & 80.87 & 80.87 & 3.51 \\
\hline
\end{tabular}




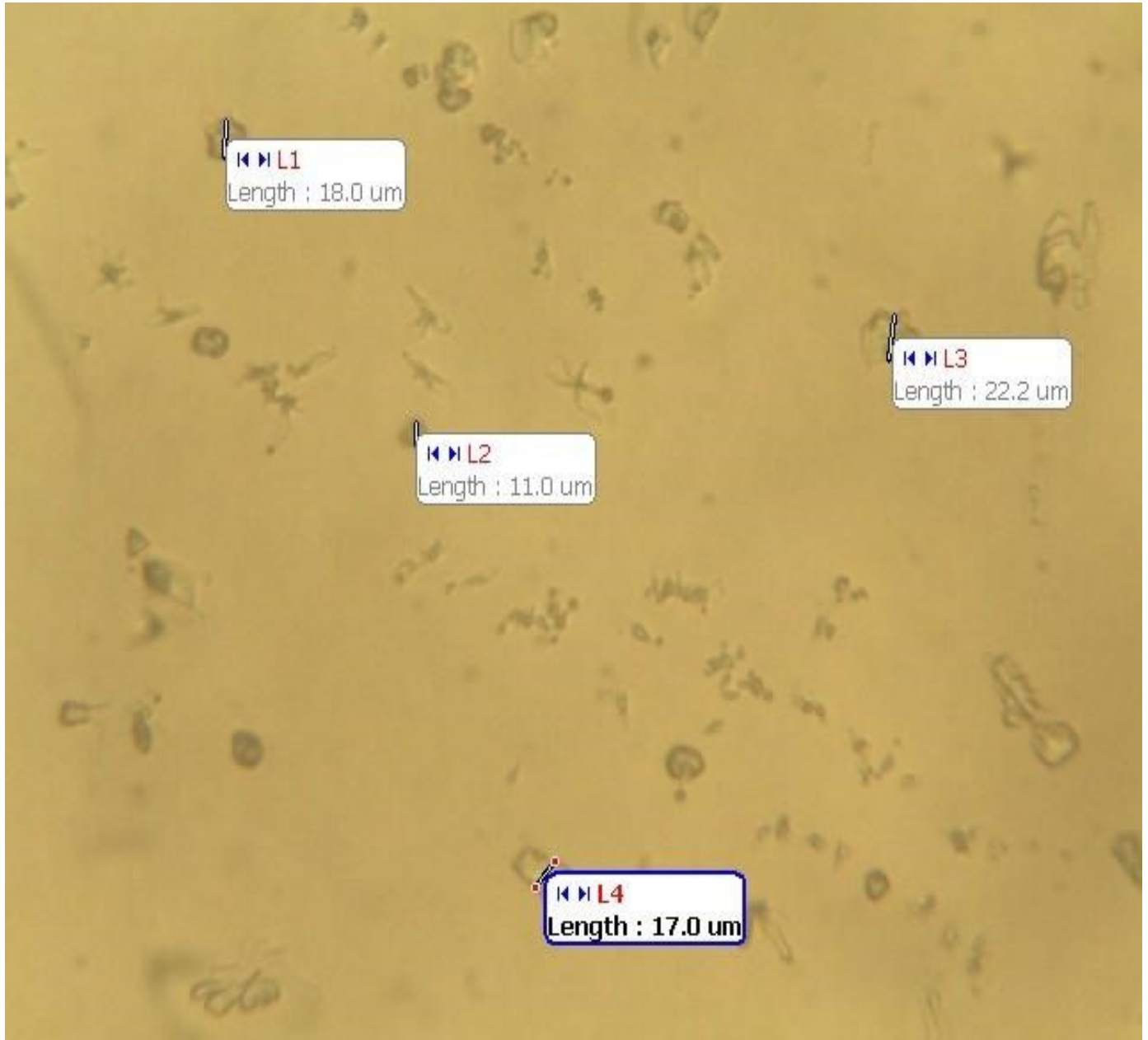

Fig. 1 Photomicrograph of formulation $B$.

Table 3 Mean inhibition zone diameter $(\mathrm{mm})$ for pure erythromycin.

\begin{tabular}{llll}
\hline Test Org. & \multicolumn{1}{c}{$\begin{array}{c}\text { Erythromycin concentrations } \\
(\mu \mathrm{g} / \mathrm{mL})\end{array}$} & Controls \\
\hline & \multicolumn{1}{c}{\begin{tabular}{c}
\multicolumn{1}{c}{ Eryth. $(30 \mu \mathrm{g} / \mathrm{mL})$} \\
E. coli
\end{tabular}} & $\begin{array}{l}\text { Sterile water } \\
(10 \mathrm{~mL} / \mathrm{kg})\end{array}$ \\
& Inhibition zone diameter $(\mathrm{mm})$ & & 0 \\
K. pneumonia & $4 \pm 0.0000$ & $4 \pm 0.0$ & 0 \\
S. aureus & $16 \pm 0.514 \pm 0.012 \pm 0.08 \pm 0.5$ & $16 \pm 0.5$ & 0 \\
B. subtilis & 0000 & $18 \pm 0.0$ & 0 \\
\hline
\end{tabular}

Table 4 Mean Inhibition Zone Diameter of Erythromycin SLMs.

\begin{tabular}{|c|c|c|c|c|c|c|c|}
\hline \multirow[t]{2}{*}{ Test Org. } & \multicolumn{5}{|c|}{$\begin{array}{l}\text { Formulated erythromycin concentrations } \\
\qquad(\mu \mathrm{g} / \mathrm{mL})\end{array}$} & \multicolumn{2}{|l|}{ Controls } \\
\hline & 191.46 & 95.73 & 47.9 & 23.93 & 11.96 & Positive & Negative \\
\hline E. coli & $10 \pm 0.0$ & $8 \pm 0.0$ & $7 \pm 0.0$ & $5 \pm 0.0$ & $2 \pm 0.0$ & $\begin{array}{l}\text { Eryth. }(30 \mu \mathrm{g} / \mathrm{mL}) \\
4 \pm 0.0\end{array}$ & $\begin{array}{l}\text { Sterile water } \\
0\end{array}$ \\
\hline K. pneumonia & $22 \pm 0.0$ & $20 \pm 0.5$ & $16 \pm 0.0$ & $14 \pm 0.5$ & $12 \pm 0.5$ & $16 \pm 0.5$ & 0 \\
\hline S. aureus & $24 \pm 0.0$ & $22 \pm 0.0$ & $20 \pm 0.0$ & $18 \pm 0.0$ & $16 \pm 0.0$ & $18 \pm 0.0$ & 0 \\
\hline B. subtilis & 0 & 0 & 0 & 0 & 0 & 0 & 0 \\
\hline
\end{tabular}

Highest concentration was chosen as not to cause turbidity in the medium. 
Table 5 Mean inhibition zone diameter ( $\mathrm{mm}$ ) for plain SLMs.

\begin{tabular}{llllll}
\hline & $6.6 \mathrm{mg} / \mathrm{mL}$ & $3.3 \mathrm{mg} / \mathrm{mL}$ & $1.65 \mathrm{mg} / \mathrm{mL}$ & $0.875 \mathrm{mg} / \mathrm{mL}$ & $0.4875 \mathrm{mg} / \mathrm{mL}$ \\
\hline E. coli & 2 & 0 & 0 & 0 & 0 \\
K. pneumonia & 4 & 0 & 0 & 0 & 0 \\
S. aureus & 4 & 2 & 0 & 0 & 0 \\
B. subtilis & 0 & 0 & 0 & 0 & 0 \\
\hline
\end{tabular}

Table 6 In vivo efficacy of the various samples.

\begin{tabular}{|c|c|c|c|c|}
\hline Groups of albino mice & $\mathrm{A}$ & $\mathrm{B}$ & $\mathrm{C}$ & $\mathrm{D}$ \\
\hline Initial E. coli count & 0 & 0 & 0 & 0 \\
\hline $\begin{array}{l}\text { E. coli count at } 24 \mathrm{~h} \text { post infection } \\
\text { (bacteremia) }\end{array}$ & $390 \pm 8$ & $420 \pm 15$ & $400 \pm 10$ & $415 \pm 11$ \\
\hline$\%$ bacteremia at $48 \mathrm{~h}$ & $103.2 \pm 10.7 \%$ & $72.9 \pm 18.4 \% *$ & NA & NA \\
\hline$\%$ bacteremia at $56 \mathrm{~h}$ & $108.4 \pm 6.9 \%$ & $58.5 \pm 12.1 \% *$ & NA & NA \\
\hline$\%$ bacteremia at $72 \mathrm{~h}$ & NA & $33.8 \pm 9.9 \%$ & NA & NA \\
\hline
\end{tabular}

Key: A = Animals that received pure erythromycin; B = Animals that received erythromycin SLMs; C $=$ Animals that received plain SLMs; $\mathrm{D}=$ Animals that received no treatment; $\mathrm{NA}=$ Not applicable, meaning death recorded in the group.

*significant at $p<0.05$.

efficient carrier systems for erythromycin as encapsulation efficiency ranged from $78.40 \%$ to $94.83 \%$. It has been shown that drug encapsulation efficiency depends on the amount of drug used. Momoh et al. reported that the efficiency of entrappment in Ibuprofen loaded beeswax and Phospholipon 90H lipospheres increased from 89.4\% to $97.9 \%$ as the ammount of Ibuprofen increased from $100 \mathrm{mg}$ to $400 \mathrm{mg}$ [17]. One other parameter that affects $\mathrm{EE} \%$ when total amount of drug used is constant is the lipid concentration. Sahu and coworkers studied the influence of the independent variable, lipid concentration, on the EE\% of erythromycin solid lipid nanoparticles and found that it had a positive effect [18]. This agrees with the findings of the present work since doubling the amount of beeswax led to a significant increase in $\mathrm{EE} \%$ from $81.21 \%$ to $94.83 \%$. This could be attributed to a favorable interaction between the hydrophobic model drug and the encapsulating lipid matrix. Some other workers working with Beeswax and carnauba wax attributed high $\mathrm{EE} \%$ to high thermal stability and efficiency of the mixing and homogenizing process [19]. Low encapsulation efficiency has been attributed to hydrophobicity of the lipid matrix, the porous nature of the lipid microparticles as well as the solubility [20]. Drug loading capacity gives the amount of drug per given weight of the microparticles. The trend for the loading capacity did not depend on entrapment efficiency.

The result of in vitro assessment of the activity of test samples using various organisms showed that erythromycin had no activity against $B$. subtilis. It is possible that the strain used is not susceptible to erythromycin as it has been shown that various strains of $B$. subtilis have varying degrees of susceptibility to erythromycin [21]. As expected, erythromycin whether formulated or not had a concentration-dependent activity against the other organisms. The ranking of the activity against the organism is $S$. aureus $>K$. pneumonia $>E$. coli. The zone of inhibition observed for the formulated erythromycin was higher than that of pure erythromycin. This may be due partly to the higher solubilization afforded by the formulation excipients and to high surface area arising from the formulation. It could also be due to the modifying effect of the surfactants on the cell membrane of the organism resulting in a faster influx of the drug. It is important to note that higher concentration of the erythromycin SLMs was needed to achieve higher activity. It is expected that in in vivo situation, much lower 
concentration may be required since some enzymes and physiological regulators required to digest the SLMs in the body are lacking in the in vitro setting. Noteworthy is also the fact that the plain SLMs had activity albeit very small. This is most probably due to the presence of beeswax, which has been shown to possess a fair level of antimicrobial activity [22], in the formulation.

The level of bacteremia in the in vivo studies increased for pure erythromycin sample while it reduced for erythromycin SLMs. The significant reduction in bacteremia in spite of the low level of activity of erythromycin against $E$. coli in vitro is an evidence of the ability of the SLMs in improving the oral bioavailability of the drug. All the animals in treatment groups 3 and 4 died before the completion of the 72 hours of treatment. These observations correlate with the result of in vitro assessment since equivalent doses of erythromycin were administered. Drug loading capacity for the SLMs was used to calculate the dose administered to animals. This observation also shows that erythromycin release from the SLMs in vivo is more efficient than in vitro.

\section{Conclusion}

Formulation of erythromycin ethyl succinate in using solid-lipid particulate matrix of beeswax markedly improved its antibacterial efficacy.

\section{Acknowledgements}

The authors wish to acknowledge the kind assistance in handling the animals by $\mathrm{Mr}$ Gugu Thaddeus, a laboratory technologist in the Department of Pharmaceutical Microbiology and Biotechnology, Faculty of Pharmaceutical Sciences of Nnamdi Azikiwe University, Awka, Nigeria.

\section{References}

[1] Kawakami, K., Yoshikawa, T., Hayashi, T., Nishihara, Y., and Masuda, K. 2002. "Microemulsion Formulation for Enhanced Absorption of Poorly Soluble Drugs II." Journal of controlled Release 81: 75-82.
[2] Amidon, G. L., Lennernäs, H., Shah, V. P., and Crison, J. R. 1995. "A Theoretical Basis for a Biopharmaceutic Drug Classification: The Correlation of in Vitro Drug Product Dissolution and in Vivo Bioavailability." Pharm. Res. 12 (3): 413-20.

[3] Pramod, R. S., Balasaheb, S. P., Shilpi, R., Sarita, S. R., and Amruta, B. V. 2014. "Approaches towards the Solubility Enhancement of Drug: A Review." World Journal of Pharmacy and Pharmaceutical Sciences 3 (4): 625-45.

[4] Attama, A. A., Momoh, M. M., and Builders, P. F. 2012. "Lipid Nanoparticulate Drug Delivery System: A Revolution in Dosage form Design and Development. In Recent Advances in Novel Drug Carrier Systems." Edited by Intech. Retrieved online on March 24, 2016 from http://cdn.intechopen.com/pdfs-wm/40253.pdf.

[5] Benameur, H. 2012. "Lipid Based Dosage Forms-An Emerging Platform for Drug Delivery." Retrieved on March 24, 2016 from http://www.scribd.com/doc/274195065/Lipid-based-Dosa ge-Forms-An-EmergingPlatform-for-Drug-Delivery-REV IEW-GOOD.

[6] Umeyor, C. E., Kenechukwu, F. K., Uronnachi, E. M., Osonwa, U. E., and Nwakile, C. D. 2012. "Solid Lipid Microparticles: An Effective Lipid Based Technology for Controlled Drug Delivery." Am. J. PharmTech Res. 2 (6): 1-18.

[7] Dalpiaz, A., Mezzena. M., Scatturin, A., and Scalia, S. 2008. "Solid Lipid Microparticles for the Stability Enhancement of the Polar Drug N6-Cyclopentyladenosine." Int. J Pharm. 355: 81-6.

[8] Garud, A. Singh, D., and Garud, N. 2012. "SLN (Solid Lipid Nanoparticles): Method, Characterization and Applications." International Current Pharmaceutical Journal 1 (11): 384-93.

[9] Fahr, A., and Liu, X. 2007. "Drug Delivery Strategies for Poorly Water-soluble Drugs." Expert Opinion on Drug Delivery 4 (4): 403-16.

[10] Muller, R. H., Mehnert, W., Lucks, J. S., Schwarz, C., Zur Muhlen, A., Meyhers, H., Freitas, C., and Ruhl, D. 1995. "SLN (Solid Lipid Nanoparticles) - An Alternative Colloidal Carrier System for Controlled Drug Delivery." Eur. J. Pharm Biopharm. 41: 62-9.

[11] Rupenagunta, A., Somasundaram, I., Ravichandiram, V., Kausalya, J., and Senthilnathan, B. 2011. "Solid Lipid Nanoparticles-A Versatile Carrier System." J. Pharm Res. 4 (7): 2069-75.

[12] Yang, S., Zhu, J., Lu, Y., Liang, B., and Yang, C. 1999. "Body Distribution of Camptothecin Solid Lipid Nanoparticles after Oral Administration." Pharm Res 16 (5): 751-7.

[13] Kohno, Y. 2002. "Pharmakokinetics and Metabolism of 
Macrolides." In Macrolide Antibiotics: Chemistry, Biology and Practice, edited by Omura, S. London: Academic press, 327-61.

[14] Wilson, J. T, and Van Boxtel, C. J. 1978. 'Pharmakokinetics of Erythromycin in Man.' Antibiot. Chemother 25: 181-203.

[15] Ginsburg, C. M. 1986. "Pharmacology of Erythromycin in Infants and Children." Pediatr. Infect. Dis. J 5: 124-9.

[16] Diab, R., Jaafar-Maalej, C., Fessi, H., and Maincent, P. 2012. "Engineered Nanoparticulate Drug Delivery Systems: The Next Frontier for Oral Administration?" AAPS Journal 14 (4): 688-702.

[17] Momoh, M. A., Kenechukwu, F. C, Gwarzo, M. S., and Builders, P. F. 2015. "Formulation and Evaluation of Ibubrofen Loaded Liposheres for Effective Oral Delivery." Dhaka Univ. J. Pharm Sci. 14 (1): 17-27.

[18] Sahu, A. K., Kumar, T., and Jain, V. 2016. "Formulation Optimization of Erythromycin Solid Lipid Nanocarrier Using Response Surface Methodology BioMed Research
International, 2014.” Retrieved online on March 24, from file:///C:/Users/Uduma/Downloads/689391.pdf.

[19] Martins, R. M., Siqueira, S., Fonseca, M. V., and Freitas, L. A. P. 2014. "Skin Penetration and Photoprotection of Topical Formulations Containing Benzophenone-3 Solid Lipid Microparticles Prepared by the Solvent-Free Spray-Congealing Technique.” J. Microencapsul 31 (7): 644-53.

[20] Mishra, H., Mishra, D., Mishra, P. K., Nahar, M., Dubey, V., and Jain, D. K. 2010. "Evaluation of Solid Lipid Nanoparticles as Carriers for Delivery of Hepatitis B Surface Antigen for Vaccination Using Subcutaneous Route." J Pharm Pharmaceut Sci 13 (4): 495-509.

[21] Beyalti, Y., Saglam, N., and Aslim, B. 2002. "Determination of Some Properties of Bacillus Isolated from Soil." Turk J Biol 26: 41-8.

[22] Al-Waili, N. S. 2005. "Mixture of Honey, Beeswax and Olive Oil Inhibits Growth of Staphylococcus Aureus and Candida Albicans." Arch Med Res. 36 (1): 10-23. 